\title{
Generalized Gaussian Quadrature Rules for Discontinuities and Crack Singularities in the Extended Finite Element Method
}

\author{
S. E. Mousavi, N. Sukumar \\ Department of Civil and Environmental Engineering, University of California \\ One Shields Avenue, Davis, CA 95616, U.S.A.
}

\begin{abstract}
New Gaussian integration schemes are presented for the efficient and accurate evaluation of weak form integrals in the extended finite element method. For discontinuous functions, we construct Gauss-like quadrature rules over arbitrarily-shaped elements in two dimensions without the need for partitioning the finite element. A point elimination algorithm is used in the construction of the quadratures, which ensures that the final quadratures have minimal number of Gauss points. For weakly singular integrands, we apply a polar transformation that eliminates the singularity so that the integration can be performed efficiently and accurately. Numerical examples in elastic fracture using the extended finite element method are presented to illustrate the performance of the new integration techniques.
\end{abstract}

Key words: partition-of-unity enrichment, numerical integration, discontinuous functions, weakly singular functions, generalized Duffy transformation, node elimination algorithm, crack growth

\footnotetext{
${ }^{*}$ Corresponding author

Email address: nsukumar@ucdavis.edu (N. Sukumar)
} 


\section{Introduction}

In partition of unity methods, special basis functions are added to the solution space to allow the inclusion of a priori knowledge about the local behavior of the solution [1-3]. In the extended finite element method (XFEM) [4-8], which is used for crack modeling without remeshing, the displacement approximation is enriched by incorporating a discontinuous function and the near-tip asymptotic fields via the framework of partition of unity. A closely-related approach to the X-FEM for modeling cracks is that due to Hansbo and Hansbo [9], and recently a few different variants on the XFEM for fracture mechanics have been proposed [10-12]. In these methods, a well-known and outstanding issue is the efficient and accurate evaluation of the entries in the stiffness matrix corresponding to the enriched degrees of freedom since the gradient of enriched basis functions contain discontinuities and/or singularities. Traditionally, these elements are partitioned into integration subcells (normally subtriangles in two dimensions) that conform to the crack geometry and higher-order Gauss quadrature is used over them for integration [5]. In this paper, we present the construction of generalized Gaussian quadrature rules that exactly integrate polynomials on either side of the crack without the need to partition the finite element. Furthermore, we adopt the generalized Duffy transformation introduced by Mousavi and Sukumar [13] to enable the efficient and accurate computation of weakly singular stiffness matrix integrals in fracture computations with the X-FEM.

The integration of discontinuous functions arises in many partition-ofunity enriched finite element applications: quasi-static crack growth $[4,5]$, dynamic fracture [14-16], cohesive crack modeling [17-19], two-phase fluid flow and fluid-structure interaction [20-23], surgical simulations [24, 25], fracture animations [26], etc. Ventura [27] showed that an equivalent polynomial function exists such that its integral gives the exact value of the 
discontinuous/non-differentiable function integrated on subcells; as a result there is no need to divide the cut element into subcells and use quadrature rules over the background cells. The coefficients of the equivalent polynomials are functions of the location of the discontinuity in the element. Tornberg [28] and Patzák and Jirásek [29] proposed regularized forms of the Heaviside function that smear the strong discontinuity over a short distance on which the discontinuous function is approximated by a smooth function. Oh et al. [30] introduce several smooth-piecewise-polynomial regularized discontinuous functions that can be used in the X-FEM. Benvenuti et al. [31] extended the method of Ventura [27] for regularized discontinuous enrichment functions. The technique presented in References $[27,31]$ is restricted to linear triangular and quadrilateral elements in two dimensions and linear tetrahedral elements in three dimensions with cracks that do not kink inside elements [32]. Holdych et al. [33] proposed a method for construction of quadrature rules over triangles and tetrahedrons (cut by a crack) by fixing the location of the integration points and allowing for variable weights. Belytschko et al. [34] showed that the weights of the quadrature points determined by Holdych et al. [33] and the values of the equivalent polynomials of Ventura [27] are related. For integrating discontinuous functions, the limitations of the aforementioned methods are that they are dependent on the element topology and due to the complexity of the algebraic expressions, crack kinks within an element can not be handled.

Xiao and Gimbutas [35] presented the node elimination technique for the construction and optimization of quadrature rules on triangles and squares for general classes of functions based on group theory and numerical optimization. Mousavi et al. [36] extended the algorithm for the construction of efficient quadrature rules over arbitrary polygons. The quadratures that are realized have desirable properties such as interiority of integration points, 
positive weights, and minimum number of points. Here, we generalize the algorithm in Reference [36] to develop quadrature rules for piecewise polynomials on finite elements that are intersected by kinked cracks.

Special conforming elements have been designed for accurate modeling of crack-tip asymptotic fields in the finite element method [37-43]. The interpolation functions of these elements are defined so that their derivatives have the desired $1 / \sqrt{r}$ crack-singularity. The integration of the stiffness matrix entries over these crack-tip elements is done using either standard Gaussian quadrature rules [37-39, 41, 43] or by means of ad hoc quadratures tailored for specific singular shape function derivatives [40, 42, 44]. These techniques can not be applied to the X-FEM where the crack-tip can lie inside an element-stiffness matrix integrands in these elements contain singular terms of $\mathcal{O}(1 / r)$ and $\mathcal{O}(1 / \sqrt{r})$. Moës et al. [5] subdivide the element containing the crack-tip into conforming triangles and use higher-order Gauss quadratures over each of them. Natarajan et al. [45] use the SchwarzChristoffel conformal mapping from an arbitrary polygon to the unit disk and then apply a midpoint rule to the mapped domain. This approach obviates the need to use two successive isoparametric mappings in polygonal finite element applications [46, 47], which simplifies the implementation; however, the number of integration points and the accuracy is of the same order as the subdivision scheme presented by Moës et al. [5].

In adaptive integration schemes [48], the point-singularity that lies inside the finite element is resolved by recursively subdividing the element into nonconforming subcells until a prescribed error tolerance is achieved. Strouboulis et al. [49] as well as Xiao and Karihaloo [50] use DECUHR [51], an adaptive integration scheme based on nonuniform subdivision of elements for integration of singular functions. Schweitzer [52] adopts a hierarchical adaptive integration technique [53] for the enrichment functions in the particle-partition 
of unity method by decomposing the integration domain into cells and using tensor-product quadrature rules over the cells. Standard Gauss quadratures implicitly approximate the singular integrand with polynomials; therefore, many integration points are needed for even modest accuracy. Béchet et al. [54] present a scheme in which first the domain is triangulated with the singularity lying at a vertex and then the integration of the singular kernel over the triangle is transformed into a smooth integration over the biunit square through a series of transformations. Although efficient in two dimensions, the mapping in Reference [54] does not readily extend to three dimensions. Park et al. [55] use the mapping of Nagarajan and Mukherjee [56] and generalize it to three dimensions for crack problems. This mapping is advantageous for integration of homogeneous functions where the integration with respect to the radial term can be carried out analytically, which reduces the dimensionality of the integral by one. However, in the finite element method, where the integrand is in general neither homogeneous nor explicitly known, this scheme has limitations. For fracture problems, Shen and Lew [12] use the integration scheme proposed by Ventura et al. [57] — Green's theorem is used to convert the volume integral to a boundary integral for enrichment functions that satisfy the equilibrium equations of elastostatics.

Laborde et al. [58] employ a rather straightforward transformation from the triangle to the unit square to remove the singularity. This approach was originally conceived by Fairweather et al. [59] in two dimensions and Duffy [60] in two and three dimensions. The Duffy mapping removes the $1 / r$ singularity and the transformed integral is amenable to tensor-product Gauss quadrature. Mousavi and Sukumar [13] have shown that the Duffy transformation is efficient for a $1 / r$ singularity, but is not as efficient for $1 / r^{\alpha}$ singularities when $\alpha \neq 1$ (e.g., crack modeling in the X-FEM where $\alpha=1 / 2$ is present). Moreover, for $1<\alpha<2$ in two dimensions $(2<\alpha<3$ in three 
dimensions), the Duffy transformation does not remove the singularity. In Reference [13], a generalized form of the Duffy transformation is introduced in which a parameter $\beta$ is judiciously selected so that the transformation leads to removal of the weak singularity. Also, in case of a fractional $\alpha$ such as $\alpha=1 / 2$, the generalized Duffy transformation permits dramatic improvements in accuracy.

The remainder of this paper is organized as follows. In Section 2, a brief review of the node elimination algorithm is presented, and the construction of quadratures for discontinuous functions is described with supportive examples. In Section 3, the generalized Duffy transformation and its use within the X-FEM is presented. Quadrature rules for discontinuous and singular functions are tested in Section 4 on static and quasi-static crack growth simulations with the X-FEM to demonstrate the benefits of using the new integration techniques. Finally, we close with a few final remarks in Section 5.

\section{Quadrature Rules for Discontinuous Functions}

\subsection{Algorithm for the construction of efficient quadrature rules}

A quadrature is a formula of the form

$$
\int_{\Omega} \omega(\mathbf{x}) f(\mathbf{x}) d \mathbf{x} \approx \sum_{i=1}^{n} w_{i} f\left(\mathbf{x}_{\mathbf{i}}\right),
$$

where $\Omega$ is the domain of integration, $f$ is an integrand defined on $\Omega$ and $\omega$ is the weight function. $\mathbf{x}_{i}$ and $w_{i}$ are called quadrature points and weights, respectively. Quadrature rules are designed so that (1) is exact for all functions in a pre-selected set $\left\{\phi_{i}\right\}_{i=1}^{m}$, such as polynomials or trigonometric functions. Different techniques have been used for construction of such quadrature rules, for instance, moment fitting equations [61] and common zeros of orthogonal polynomials [62]. Recently, Xiao and Gimbutas [35] combined group theory and numerical optimization to present a new algorithm for the construction 
of efficient quadratures. They showed that given the basis functions and the domain of integration, the points and weights of a quadrature can be obtained by solving the following system of equations:

$$
\left(\begin{array}{c}
\int_{\Omega} \omega(\mathbf{x}) \phi_{1}(\mathbf{x}) d \mathbf{x} \\
\int_{\Omega} \omega(\mathbf{x}) \phi_{2}(\mathbf{x}) d \mathbf{x} \\
\vdots \\
\int_{\Omega} \omega(\mathbf{x}) \phi_{m}(\mathbf{x}) d \mathbf{x}
\end{array}\right)=\left(\begin{array}{cccc}
\phi_{1}\left(\mathbf{x}_{1}\right) & \phi_{1}\left(\mathbf{x}_{2}\right) & \ldots & \phi_{1}\left(\mathbf{x}_{n}\right) \\
\phi_{2}\left(\mathbf{x}_{1}\right) & \phi_{2}\left(\mathbf{x}_{2}\right) & \ldots & \phi_{2}\left(\mathbf{x}_{n}\right) \\
\vdots & & & \\
\phi_{m}\left(\mathbf{x}_{1}\right) & \phi_{m}\left(\mathbf{x}_{2}\right) & \ldots & \phi_{m}\left(\mathbf{x}_{n}\right)
\end{array}\right)\left(\begin{array}{c}
w_{1} \\
w_{2} \\
\vdots \\
w_{n}
\end{array}\right)
$$

Equation (2) is a nonlinear system of equations that can be solved using Newton's least squares method. Xiao and Gimbutas [35] introduced the notion of optimizing the obtained quadrature rule by eliminating one of the points of the quadrature and solving (2) again. Once a solution, i. e., $\left\{\mathbf{x}_{i}, w_{i}\right\}_{i=1}^{n}$, is available it implies that an $n$-point quadrature rule has been constructed for the integration of the basis functions $\left\{\phi_{i}\right\}_{i=1}^{m}$ in $\Omega$. Now, the integration point that contributes the least is ascertained by computing the so-called significance factor $[35,36]$ :

$$
s_{j}=w_{j} \sum_{i=1}^{m} \phi_{i}^{2}\left(\mathbf{x}_{j}\right), \quad s_{k}=\min s_{j}, \quad(j=1,2, \ldots, n)
$$

and the weight $w_{k}$ is set to zero, and hence $n-1$ quadrature weights are now non-zero. The set $\left\{\mathbf{x}_{1}, w_{1}, \mathbf{x}_{2}, w_{2}, \ldots, \mathbf{x}_{k-1}, w_{k-1}, \mathbf{x}_{k+1}, w_{k+1}, \ldots, \mathbf{x}_{n}, w_{n}\right\}$ is then an approximate solution to (2). On starting from this approximate solution and solving (2) once again, a $(n-1)$-point quadrature rule is produced. This procedure (node elimination algorithm) is repeated until Newton's method fails to converge. Wandzura and Xiao [63] and Xiao and Gimbutas [35] applied this algorithm to triangles and squares for polynomial basis functions of very high orders. The node elimination algorithm is flexible and is not restricted to specific domain shapes nor classes of functions. Also, features such as symmetries in the domain of integration can be exploited by constructing quadrature rules on the symmetry group of the domain. 
Mousavi et al. [36] used the node elimination algorithm for the construction of efficient quadrature rules on convex and concave polygons.

\subsection{The node elimination algorithm for discontinuous functions}

In this paper, we use the node elimination algorithm for the construction of quadrature rules for discontinuous functions within the extended finite element method. In the X-FEM, a crack discontinuity that fully cuts an element is modeled by enriching the finite element space by a discontinuous (generalized Heaviside) function. The enriched approximation for the displacement field is $[5]$ :

$$
\mathbf{u}^{h}(\mathbf{x})=\sum_{I \in \mathcal{N}} N_{I}(\mathbf{x}) \mathbf{u}_{I}+\sum_{I \in \mathcal{N}_{\Gamma}} N_{I}(\mathbf{x}) H(\mathbf{x}) \mathbf{a}_{I},
$$

where $\mathcal{N}$ is the set of finite element nodes, $\mathcal{N}_{\Gamma}$ is the set of nodes whose shape function support is cut by the crack interior, $N_{I}(\mathbf{x})$ is the finite element shape function and $\mathbf{u}_{I}$ and $\mathbf{a}_{I}$ are the unknown nodal coefficients corresponding to the classical and Heaviside degrees of freedom, respectively. The generalized Heaviside function $H(\mathbf{x})$ is equal to +1 above the crack and -1 below the crack. Alternatively, a shifted Heaviside function can be used [14]. The discrete system of linear equations is obtained by substituting the trial and test functions in the weak form for linear elasticity. For the sake of brevity, we omit the derivation of the discrete equations and refer the reader to Reference [64]. The element stiffness matrix for an enriched element is:

$$
\mathbf{K}_{e}=\left[\begin{array}{cc}
\mathbf{k}_{u u} & \mathbf{k}_{u a} \\
\mathbf{k}_{a u} & \mathbf{k}_{a a}
\end{array}\right]=\int_{\Omega_{e}}\left[\begin{array}{cc}
\mathbf{B}_{u}^{T} \mathbf{D} \mathbf{B}_{u} & H \mathbf{B}_{u}^{T} \mathbf{D} \mathbf{B}_{u} \\
H \mathbf{B}_{u}^{T} \mathbf{D} \mathbf{B}_{u} & H^{2} \mathbf{B}_{u}^{T} \mathbf{D} \mathbf{B}_{u}
\end{array}\right] d \Omega,
$$

where $\mathbf{D}$ is the material moduli matrix and $\mathbf{B}_{u}$ is the matrix of finite element shape function derivatives. For an element with nel nodes, $\mathbf{B}_{u}$ is defined as

$$
\mathbf{B}_{u}=\left[\begin{array}{ccccccc}
N_{1, x} & 0 & N_{2, x} & 0 & \ldots & N_{n e l, x} & 0 \\
0 & N_{1, y} & 0 & N_{2, y} & \ldots & 0 & N_{n e l, y} \\
N_{1, y} & N_{1, x} & N_{2, y} & N_{2, x} & \ldots & N_{n e l, y} & N_{n e l, x}
\end{array}\right] .
$$


In order to use the node elimination algorithm for the construction of discontinuous quadrature rules it is beneficial to examine the kernel of the integral in (4). The terms $\mathbf{B}_{u}^{T} \mathbf{D} \mathbf{B}_{u}$ and $H^{2} \mathbf{B}_{u}^{T} \mathbf{D} \mathbf{B}_{u}$ are composed of polynomials on triangular and rectangular elements and are not discontinuous inside an element, whereas the term $H \mathbf{B}_{u}^{T} \mathbf{D B}_{u}$ is composed of polynomials multiplied by the Heaviside function with a discontinuity across the crack. On setting $\omega(\mathbf{x})=H(\mathbf{x})$ and $\left\{\phi_{i}(\mathbf{x})\right\}_{i=1}^{m}=\mathbf{P}_{d}(\mathbf{x})$, where $\mathbf{P}_{d}(\mathbf{x})$ stands for all bivariate polynomials up to order $d$ and solving (2), the resulting set $\left\{\mathbf{x}_{i}, w_{i}\right\}_{i=1}^{n}$ is a quadrature rule that can integrate $H \mathbf{B}_{u}^{T} \mathbf{D B}_{u}$ exactly over the element. In other words, once convergence is attained, an $n$-point quadrature rule is produced that can integrate the selected polynomials multiplied by the Heaviside function, and is specifically designed for the cracked element. The key issue in the above procedure is the evaluation of the left-hand-side of (2), which contains the integration of functions of the form $H(\mathbf{x}) p(\mathbf{x})$ over the cracked element, where $p(\mathbf{x})$ are bivariate polynomial basis functions that are known analytically. In our implementation of the algorithm, since the domain of integration, i.e., the cracked element, can be described by analytic curves (piecewise straight lines in this study), we evaluate the integrals analytically over the two partitions above and below the crack without the need to further subdivide them into triangles. Since the basis functions are homogeneous, the integration can also be carried out numerically without partitioning the cracked element into triangles, for instance by applying the method of Lasserre $[65,66]$. In a crack propagation simulation, the enrichment changes only in the vicinity of the crack-tip, and the enrichment in the elements that are fully cut by the crack remain unchanged. Therefore, the discontinuous quadrature that is built in this process is saved so that it can be reused during subsequent crack growth steps. The need and merits of constructing the generalized Gaussian quadratures for elements that are 
fully cut is particularly noteworthy in applications such as mesh-independent modeling of intergranular and transgranular fracture in polycrystalline microstructures, where the crack can assume a tortuous path [67].

\subsection{Numerical examples}

The algorithm for construction of quadrature rules and the node elimination algorithm is implemented in $\mathrm{MATLAB}^{\mathrm{TM}}$. Figure 1 shows a few examples of quadrature rules constructed for triangular, quadrilateral and hexagonal elements with different crack geometries. Figures 1a to 1d show quadrature rules for integration of functions of the form $p(\mathbf{x}) H(\mathbf{x})$ where $p(\mathbf{x})$ can be any polynomial of total order 2 with respect to $\mathbf{x}$ and $H(\mathbf{x})$ is a discontinuous function. Figures 1e to 11 show similar results for higher order polynomials. This algorithm is not restricted by the element topology or the crack geometry; crack kinking inside an element can also be taken into account. As illustrated in Figures 1d, $1 \mathrm{~h}$ and 1l, the discontinuous quadrature rules can also be applied to the extended finite element fracture computations on polygonal elements [47]. In Figures $1 \mathrm{~m}-1 \mathrm{p}$, the locations of the integration points are shown for elements that are cut by two cracks; in the quadrature algorithm, the weight function $\omega(\mathbf{x})$ takes on the values $1,0,-1$ in the three regions that are separated by the cracks. The quadrature points in elements that are intersected by a branched crack $[7,68]$ are marked in Fig. 2. Since all the above quadrature rules are exact to within machine precision by construction, we do not study their accuracy here. We compare the number of evaluation points required for the integration of discontinuous functions shown in Figures 1 and 2 with standard quadrature rules over triangles versus our discontinuous quadratures in Table 1. Dunavant [69] presents 3-, 4and 6-point quadrature rules for integration of quadratic, cubic and quartic polynomials over triangles. In each case the node elimination algorithm pro-

duces very efficient quadrature rules that can be saved and reused over the 
same element with just a one-time cost of calculating the quadrature points and weights, provided that the element geometry and how it is cut by the cracks remains fixed.

\section{Quadrature Rules for Weakly Singular Functions}

\subsection{Generalized Duffy transformation}

Duffy transformation $[59,60]:(u, v) \rightarrow(x, y): x=u, y=x v=u v$, maps the standard triangle (Fig. 3a) to a unit square (Fig. 3b) and as a result, eliminates singularities of the form $1 / r$. The singular integrand over the standard triangle is transformed into a smooth function over the unit square that can be integrated with fewer integration points [58, 60], e.g., by using a tensor-product rule. Integration of any singular function over an element with the singularity inside it can be carried out by following these steps: triangulate the element so that the singularity occurs at one vertex of all triangles, map each one of the triangles to the standard one and evaluate the integration by using Duffy transformation and a standard Gauss quadrature rule over the unit square. This scheme has been used for integration of stiffness matrix entries in the X-FEM where the shape function space is enriched with near-tip functions that have singular derivatives [58]. Mousavi and Sukumar [13] presented a generalized form of the Duffy transformation: $(u, v) \rightarrow(x, y): x=u^{\beta}, y=x v=u^{\beta} v$, which maps the same standard triangle of Duffy transformation (Fig. 3a) to the unit square as follows:

$$
\mathcal{I}=\int_{0}^{1} \int_{0}^{x} \frac{f(x, y)}{\left(x^{2}+y^{2}\right)^{\alpha / 2}} d x d y=\int_{0}^{1} \int_{0}^{1} \frac{f\left(u^{\beta}, u^{\beta} v\right)}{\left(1+v^{2}\right)^{\alpha / 2}} \beta u^{2 \beta-1-\alpha \beta} d u d v
$$

The authors showed that although Duffy transformation is very effective for integration of functions with $1 / r$ singularity, it does not lead to better accuracy for integrating $1 / r^{\alpha}$ singularities when $\alpha \neq 1$, including $\alpha=1 / 2$ that arises in crack analysis in isotropic media. The deficiency in the accurate 

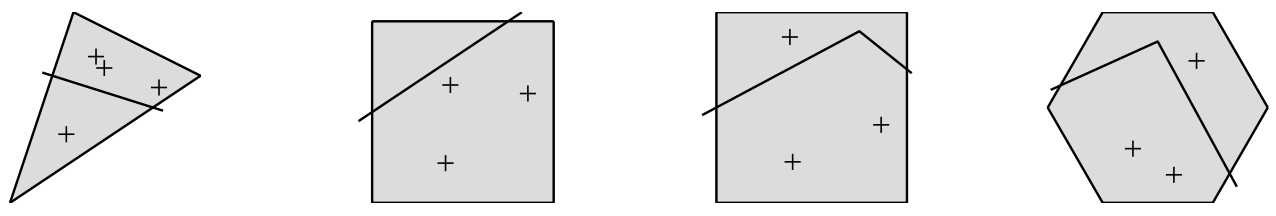

(a) $n s p=4$

(b) $n s p=3$

(c) $n s p=3$

(d) $n s p=3$
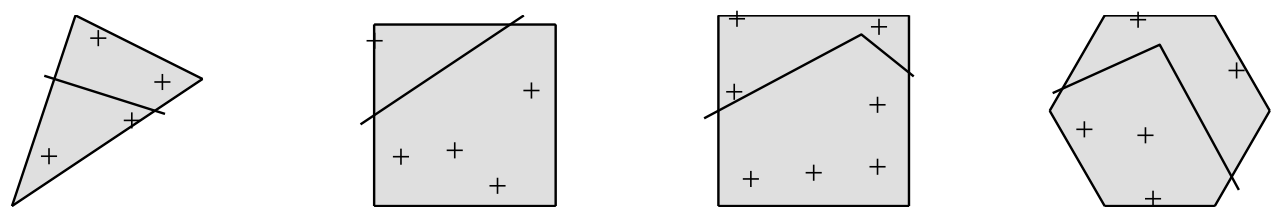

(e) $n s p=4$

(f) $n s p=5$

(g) $n s p=7$

(h) $n s p=5$
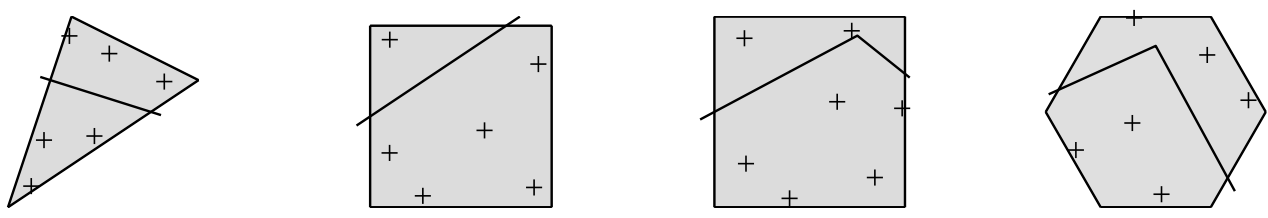

(i) $n s p=6$

(j) $n s p=6$

(k) $n s p=7$

(l) $n s p=6$
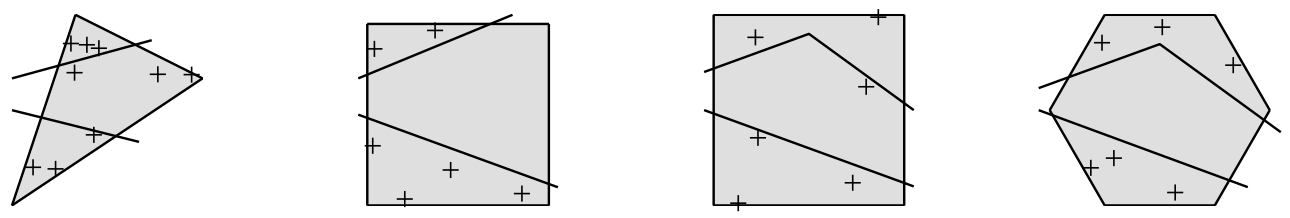
(m) $n s p=9$
(n) $n s p=6$
(o) $n s p=6$
(p) $n s p=6$

Fig. 1: Quadrature points for discontinuous functions. Element is cut by one crack in a-l and by two cracks in $\mathrm{m}-\mathrm{p}$. (a-d) quadratic precision; (e-h) cubic precision; (i-l) quartic precision; and (m-p) quartic precision (two cracks). 
Table 1: Comparison of number of integration points for integrating discontinuous functions.

\begin{tabular}{cccc}
\hline \multirow{2}{*}{$\begin{array}{c}\text { Case } \\
\text { (fig. no. })\end{array}$} & Partitioning & \multicolumn{2}{c}{ Discontinuous quadratures } \\
\cline { 2 - 3 } $1(\mathrm{a})$ & 7 & 21 & $n s p$ \\
$1(\mathrm{~b})$ & 8 & 24 & 4 \\
$1(\mathrm{c})$ & 26 & 78 & 3 \\
$1(\mathrm{~d})$ & 34 & 102 & 3 \\
\hline $1(\mathrm{e})$ & 7 & 28 & 3 \\
$1(\mathrm{f})$ & 8 & 32 & 4 \\
$1(\mathrm{~g})$ & 26 & 104 & 5 \\
$1(\mathrm{~h})$ & 34 & 136 & 7 \\
\hline $1(\mathrm{i})$ & 7 & 42 & 5 \\
$1(\mathrm{j})$ & 8 & 48 & 6 \\
$1(\mathrm{k})$ & 26 & 156 & 6 \\
$1(\mathrm{l})$ & 34 & 204 & 7 \\
\hline $1(\mathrm{~m})$ & 19 & 114 & 6 \\
$1(\mathrm{n})$ & 26 & 156 & 9 \\
$1(\mathrm{o})$ & 50 & 300 & 6 \\
$1(\mathrm{p})$ & 52 & 312 & 6 \\
\hline $2(\mathrm{a})$ & 31 & 186 & 6 \\
$2(\mathrm{~b})$ & 38 & 228 & 6 \\
$2(\mathrm{c})$ & 46 & 276 & 6 \\
\hline
\end{tabular}

${ }^{a}$ Partitioning is done as in Reference [64], where the polygons above and below the crack are divided into triangles by connecting the vertices of the element to the center of each polygon. Although this technique is not the most efficient method of partitioning, it provides a means to realize automatic subdivision of cracked elements. 


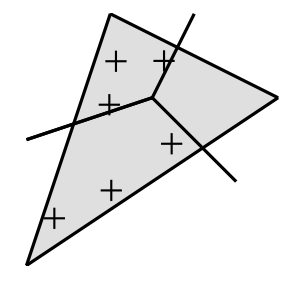

(a) $n s p=6$

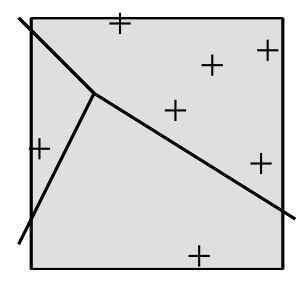

(b) $n s p=7$

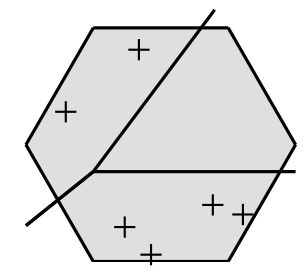

(c) $n s p=6$

Fig. 2: Quadrature points in an element with a branched crack (quartic precision).

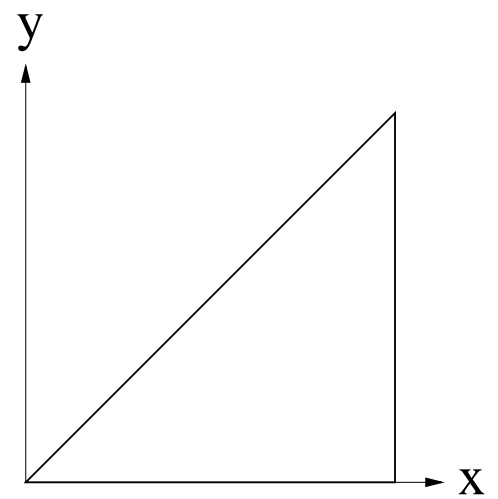

(a) $0 \leq x \leq 1,0 \leq y \leq x$

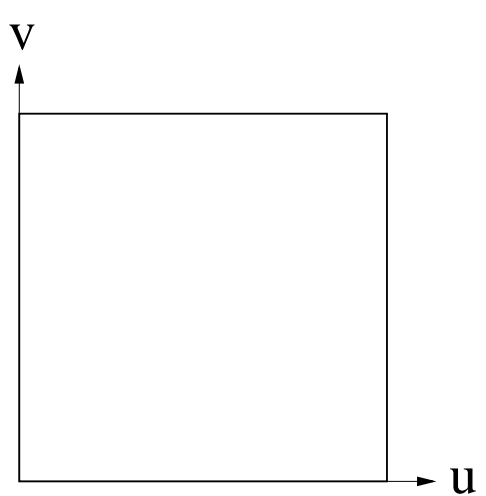

(b) $0 \leq u, v \leq 1$

Fig. 3: Duffy and generalized Duffy transformations. (a) standard triangle and (b) unit square 
evaluation of these singular integrals is due to the presence of fractional exponents of $u$ in the transformed kernel [13]. However, the parameter $\beta$ in the generalized transformation can be determined judiciously according to the exponent $\alpha$ so that the kernel after transformation remains a polynomial with respect to $u$. For example, for $\alpha=\{1,1 / 2\}, \beta=\{1,2\}$ is suggested, respectively. In addition, it is shown that increasing $\beta$ results in a larger exponent of $u$ in the transformed kernel, which necessitates the use of a slightly higher-order quadrature rule.

The generalized Duffy transformation was tested within a partition-ofunity enriched finite element method for the solution of the well-known benchmark problem of the $L$-shaped domain with a re-entrant corner [13]. The corner singularity required the evaluation of stiffness matrix entries that had $1 / r^{\alpha}$ singularities $(\alpha=1 / 3,2 / 3,4 / 3)$, and hence $\beta=3$ was adopted. $\mathrm{Nu}^{-}$ merical results were presented that demonstrated the remarkable improvement in the accuracy and convergence rate over standard quadratures or an adaptive integration scheme [49]. In this study, we apply the generalized Duffy transformation for the integration of stiffness matrix entries in the crack-tip element that contains the near-tip enrichment functions. Section 3.2 describes the application of the transformation for the integration of such functions in the X-FEM.

\subsection{Numerical examples}

The displacement approximation in the X-FEM for elements that are enriched with the near-tip functions is [5]:

$$
\mathbf{u}^{h}(\mathbf{x})=\sum_{I \in \mathcal{N}} N_{I}(\mathbf{x}) \mathbf{u}_{I}+\sum_{I \in \mathcal{N}_{\Lambda}} N_{I}(\mathbf{x}) \sum_{\alpha=1}^{4} \psi_{\alpha}(\mathbf{x}) \mathbf{b}_{I}^{\alpha}
$$

where $\mathcal{N}$ is the set of finite element nodes, $\mathcal{N}_{\Lambda}$ is the set of nodes enriched by near-tip enrichment functions, $\left\{\psi_{\alpha}\right\}_{\alpha=1}^{4}$ are the near-tip enrichment functions 
in isotropic elasticity (see Reference [4]):

$$
\left\{\psi_{\alpha}(\mathbf{x})\right\}_{\alpha=1}^{4}=\left\{\sqrt{r} \sin \frac{\theta}{2}, \sqrt{r} \cos \frac{\theta}{2}, \sqrt{r} \sin \theta \sin \frac{\theta}{2}, \sqrt{r} \sin \theta \cos \frac{\theta}{2}\right\},
$$

and $\mathbf{b}_{I}^{\alpha}$ are the associated enriched degrees of freedom. The element stiffness matrix for an element enriched with near-tip functions can be written as:

$$
\mathbf{K}_{e}=\left[\begin{array}{cc}
\mathbf{k}_{u u} & \mathbf{k}_{u b} \\
\mathbf{k}_{b u} & \mathbf{k}_{b b}
\end{array}\right]=\int_{\Omega_{e}}\left[\begin{array}{cc}
\mathbf{B}_{u}^{T} \mathbf{D B}_{u} & \mathbf{B}_{u}^{T} \mathbf{D B}_{b} \\
\mathbf{B}_{b}^{T} \mathbf{D B}_{u} & \mathbf{B}_{b}^{T} \mathbf{D B}_{b}
\end{array}\right] d \Omega
$$

where $\mathbf{B}_{u}$ and $\mathbf{B}_{b}$ are the matrix of shape function derivatives corresponding to the classical and near-tip enrichment functions, respectively. The entries of $\mathbf{B}_{b}$ are calculated using the chain rule. The classical shape function derivatives belong to the space of polynomials for triangular and rectangular elements, but the near-tip enrichment functions depend on $r$ and $\theta$ (polar coordinates in the crack-tip coordinate system) and as a result the stiffness matrix entries corresponding to these enrichment functions are generally of one of the two forms: $\{p(\mathbf{x}) f(\theta) / r\}$ or $\{p(\mathbf{x}) f(\theta) / \sqrt{r}\}$.

Since $r$ is the distance from the crack-tip and integration is done over the element containing the crack-tip, the kernels of integration have $1 / r$ and $1 / \sqrt{r}$ singularities. As an example, we evaluate two entries of the element stiffness matrix containing the near-tip enrichment functions: $k_{11}$ where the subscript 1 refers to the enriched degree of freedom of an element for the neartip function $\psi_{1}=\sqrt{r} \sin \frac{\theta}{2}$ in the $x$-direction; and $k_{21}$ where the subscript 2 refers to the classical degree of freedom of the same node in the $x$-direction. Equation (10) shows an expansion of the kernels for these integrations, where 
the subscript $i=1,2$ refers to the $i$ th column of the matrix of derivatives:

$$
\begin{aligned}
& k_{11}=\int_{\Omega_{e}} \mathbf{B}_{1}^{T} \mathbf{D B}_{1} d V=\int_{\Omega_{e}} \nabla\left(N_{1} \psi_{1}\right) \cdot \nabla\left(N_{1} \psi_{1}\right) d V \\
& =\int_{\Omega_{e}}\left[\begin{array}{lll}
\left(N_{1} \psi_{1}\right)_{, x} & 0 & \left(N_{1} \psi_{1}\right)_{, y}
\end{array}\right]\left[\begin{array}{c}
\left(N_{1} \psi_{1}\right)_{, x} \\
0 \\
\left(N_{1} \psi_{1}\right)_{, y}
\end{array}\right] d V \\
& =\int_{\Omega_{e}}\left[\left(N_{1} \psi_{1}\right)_{, x}^{2}+\left(N_{1} \psi_{1}\right)_{, y}^{2}\right] d V
\end{aligned}
$$

and

$$
\begin{aligned}
k_{21} & =\int_{\Omega_{e}} \mathbf{B}_{2}^{T} \mathbf{D B}_{1} d V=\int_{\Omega_{e}} \nabla N_{1} \cdot \nabla\left(N_{1} \psi_{1}\right) d V \\
& =\int_{\Omega_{e}}\left[\begin{array}{lll}
N_{1, x} & 0 & N_{1, y}
\end{array}\right]\left[\begin{array}{c}
\left(N_{1} \psi_{1}\right)_{, x} \\
0 \\
\left(N_{1} \psi_{1}\right)_{, y}
\end{array}\right] d V \\
& =\int_{\Omega_{e}}\left[N_{1, x}\left(N_{1} \psi_{1}\right)_{, x}+N_{1, y}\left(N_{1} \psi_{1}\right)_{, y}\right] d V
\end{aligned}
$$

where $N_{1}$ is the finite element shape function of the first node of the element and a comma denotes the derivative with respect to the indicated spatial coordinate. Clearly, the integrand in $k_{11}$ has a $1 / r$ singularity whereas the integrand in $k_{21}$ has a $1 / \sqrt{r}$ singularity. In the derivation of (10), we have assumed the constitutive matrix to be the identity without loss of generality since $\mathbf{D}$ is a constant matrix in linear elastic fracture mechanics.

First, the integration is done on the biunit square with two crack configurations: crack-tip at the center of the element (Fig. 4a) and crack-tip very close to one of the nodes (Fig. 4d). For the sake of integration, we subdivide the element into five triangles by connecting the crack-tip to the nodes of the element and also the intersection of the crack and the element edge. Different integration schemes are compared: tensor-product over the triangles by collapsing one of the nodes of a square, efficient Gaussian quadrature rules 
over the triangle [69], Duffy transformation $(\beta=1)$, and the generalized Duffy transformation $(\beta=2)$. In Fig. $4 \mathrm{~b}$, which is for the evaluation of $k_{11}$ with a $1 / r$ singularity, both the Duffy transformation $(\beta=1)$ and the generalized Duffy transformation $(\beta=2)$ exhibit better performance than standard Gaussian quadrature rules and attain an accuracy of $10^{-6}$ with a $6 \times 6$ quadrature over each triangle. However, as can be observed in Fig. 4c, for the evaluation of $k_{21}$ with a $1 / \sqrt{r}$ singularity, the Duffy transformation $(\beta=1)$ is comparable in accuracy to standard Gauss quadrature, whereas the generalized Duffy transformation $(\beta=2)$ shows remarkable improvement in accuracy as in the case for $k_{11}$. Figures $4 \mathrm{e}$ and $4 \mathrm{f}$ show similar trends when the crack-tip is close to one of the nodes of the element. The integration accuracy is, however, generally lower in this case due to the presence of ill-shaped triangles that result from subdivision. Figures $4 \mathrm{~g}$ to $4 \mathrm{l}$ show similar results for an arbitrary quadrilateral and Fig. 5 is for the integration on a linear triangular element.

\subsection{Combination of the generalized Duffy transformation and the node elim-} ination algorithm

In Section 2.2, the node elimination algorithm to construct quadrature rules for discontinuous functions over the cracked elements is described. The generalized Heaviside function $H(\mathbf{x})$ is used as the weight function in the discontinuous quadrature rules. The same approach is now adopted to illustrate the construction of quadrature rules for $1 / r^{\alpha}$ singularities. We set $\omega(\mathbf{x})=1 / r^{\alpha}$ as the weight function to construct quadrature rules, which bears similarity to the approach presented by Haegemans [70]. As in the case of quadratures for discontinuous functions, the basis functions of the singular quadratures $\left\{\phi_{i}\right\}_{i=1}^{m}$ are selected as bivariate polynomials in two dimensions. Fig. 6 shows singular quadrature rules for a biunit square and an arbitrary quadrilateral for singularities $\alpha=1$ and $\alpha=1 / 2$ with precisions 


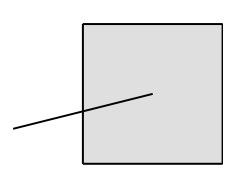

(a)

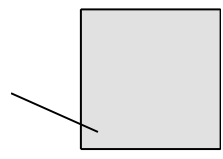

(d)

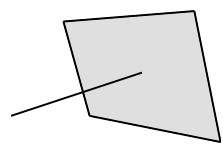

(g)

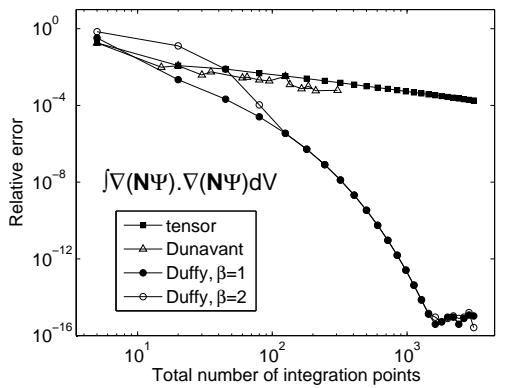

(b)

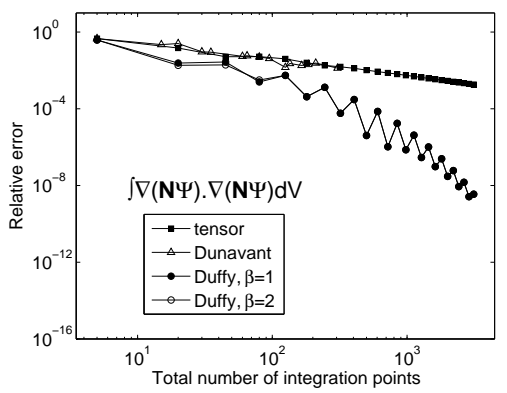

(e)

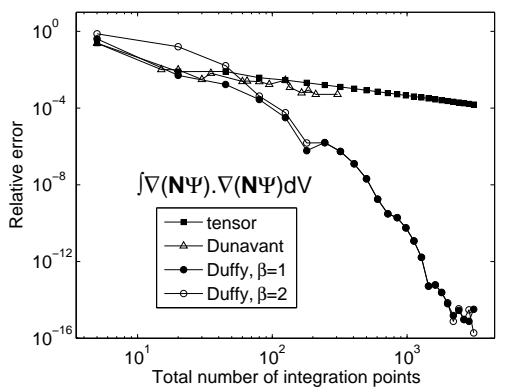

(h)

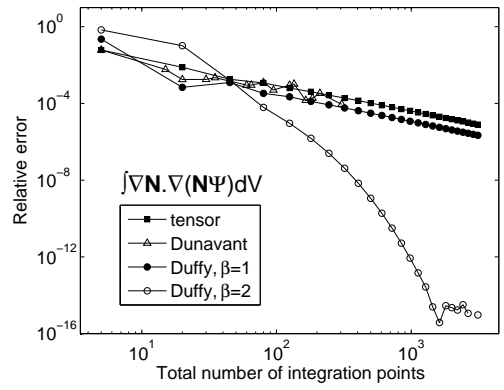

(c)

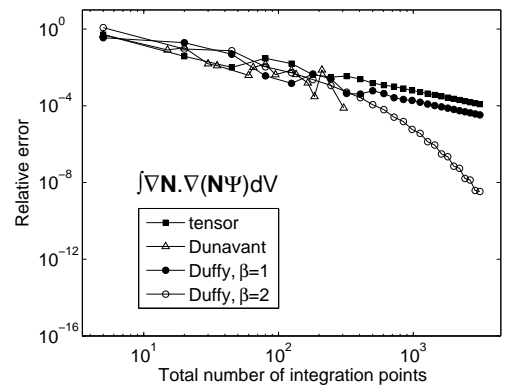

(f)

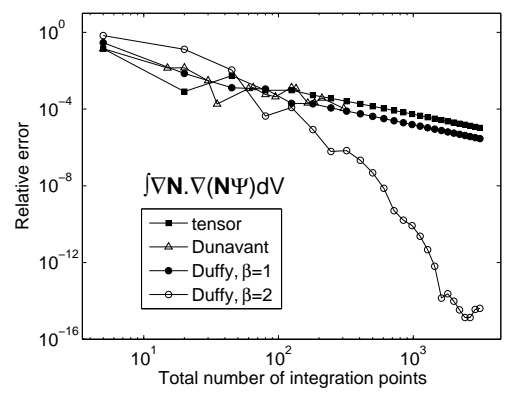

(i) 


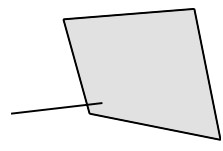

(j)

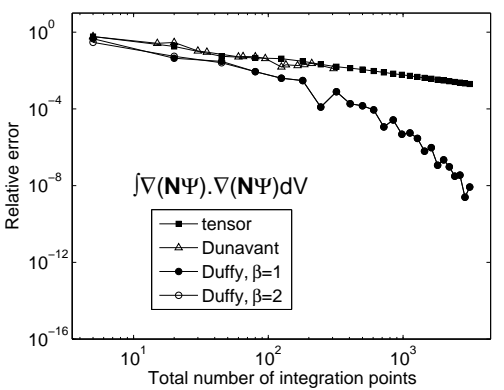

(k)

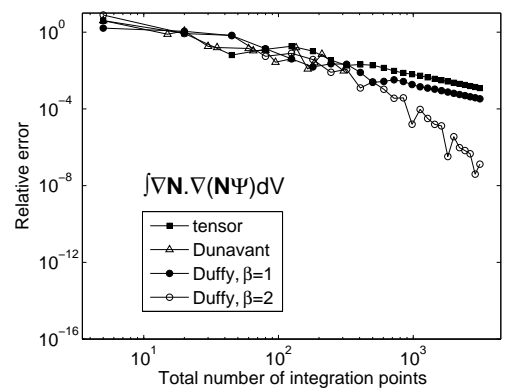

(1)

Fig. 4: Convergence of $k_{11}$ and $k_{21}$ over quadrilateral elements with different crack-tip positions.

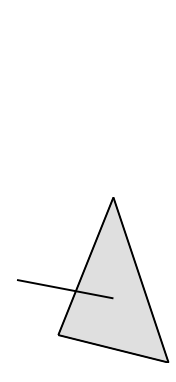

(a)

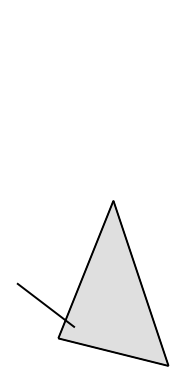

(d)

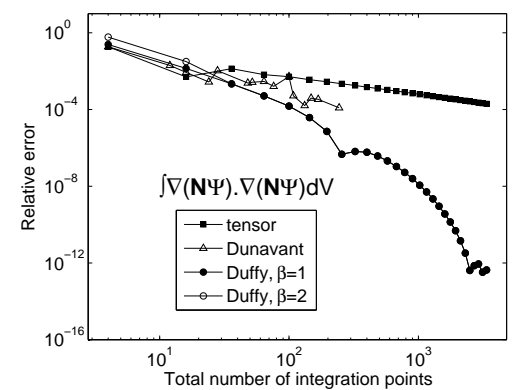

(b)

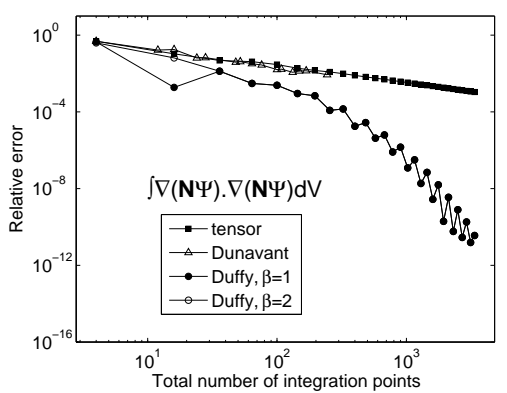

(e)

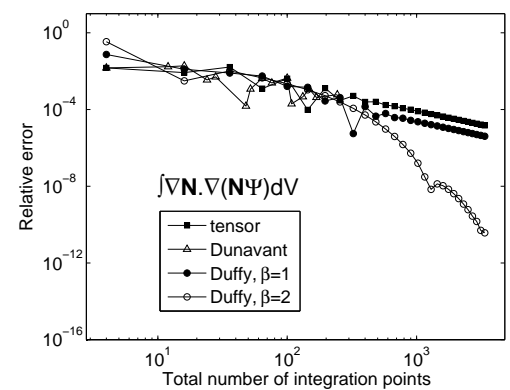

(c)

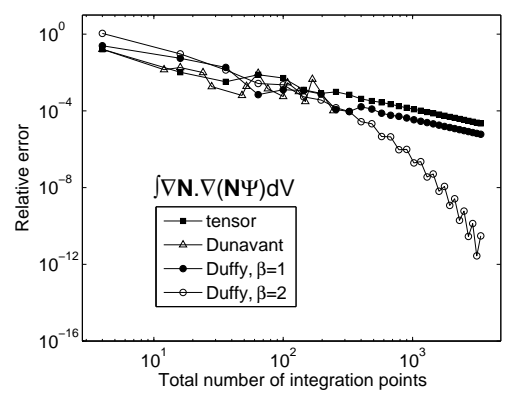

(f)

Fig. 5: Convergence of $k_{11}$ and $k_{21}$ over a triangular element with different crack-tip positions. 
of orders 2 and 5. To construct these quadrature rules, the left-hand-side of (2) is calculated by dividing the element into four triangles by connecting the source point to the vertices of the element so that the singularity lies at the vertex of all the four triangles. Then, the left-hand-side integrations are calculated using $10 \times 10$ generalized Duffy quadrature rules with the appropriate $\beta$ over each triangle. For example, the 3-point quadrature shown in Fig. 6b is equivalent to the 400 integration points over the entire element for $\alpha=1 / 2$ and $\beta=2$, and yields the same accuracy.

\subsection{Integration error in the generalized Duffy transformation}

The integration of singular functions over the standard triangle (Fig. 3a) is done by mapping the triangle to the unit square (Fig. 3b). Any arbitrary triangle with a vertex singularity is first translated so that the singularity is moved to the origin and then an affine map to the standard triangle is used: $x=a X+b Y$ and $y=c X+d Y$, where $(X, Y)$ is the physical coordinate system containing the arbitrary triangle and $(x, y)$ is the plane of the standard triangle. Equivalently, given an arbitrary triangle $R$ having one vertex at the origin, there exists an affine transformation $\mathbf{A}$ that takes the standard triangle to $R$ [71]. The transformation $\mathbf{A}$ can be used to map the points of a rule over the standard triangle to $R$. The adverse effect of the mapping is that it makes the denominator of (6) more complicated since $1+v^{2}$ changes to $1+\xi v+\eta v^{2}$, where $\xi$ and $\eta$ are functions of the coordinates of the vertices of the arbitrary triangle. After the transformation, standard Gauss quadrature rule is used in the $u$ and $v$ directions and as a result the irrational kernel with respect to $v$, i.e., $g(v) /\left(1+\xi v+\eta v^{2}\right)^{\alpha / 2}$, must be approximated by polynomials. This can reduce the accuracy especially for ill-shaped triangles.

To further examine the effect of the affine mapping on the accuracy of the integration, we fix the vertices $(0,0)$ and $(1,0)$ of a triangle and move the third vertex $(x, y)$ in the plane. The singularity is assumed to be located 


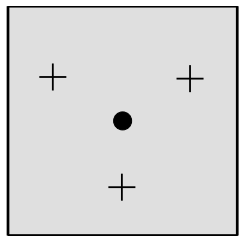

(a) $n s p=3, \alpha=1$

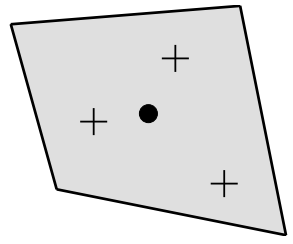

(c) $n s p=3, \alpha=1$

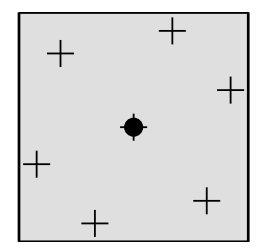

(e) $n s p=7, \alpha=1$

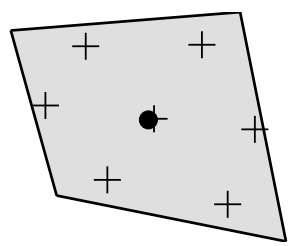

(g) $n s p=7, \alpha=1$

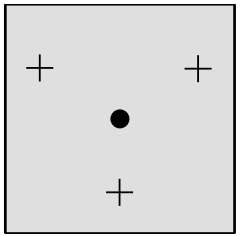

(b) $n s p=3, \alpha=1 / 2$

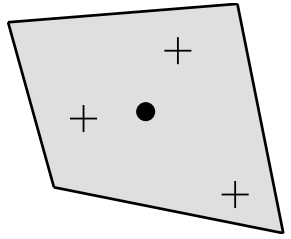

(d) $n s p=3, \alpha=1 / 2$

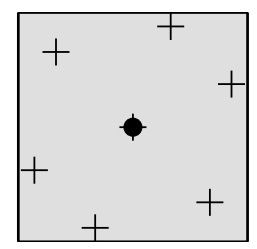

(f) $n s p=7, \alpha=1 / 2$

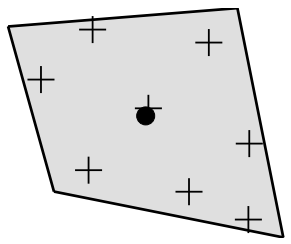

(h) $n s p=8, \alpha=1 / 2$

Fig. 6: Quadrature points $(+)$ for functions with $1 / r^{\alpha} \operatorname{singularity}(\bullet)$. (a-d) quadratic precision; and (e-h) quintic precision. 
at the origin. For each position of the third vertex, we calculate $\int_{\Omega} 1 / r^{\alpha} d A$ using the generalized Duffy transformation, where $\Omega$ is the triangle formed from the three vertices. A constant in the numerator of the integrand is used since as shown in Reference [13], the integration of a constant term has a larger error than the integration of bivariate polynomials $x^{i} y^{j}, i+j>0$. This is so since the terms $x$ and $y$ have a polar dependence and hence cause a milder singularity. The contours of the relative error for different values of $\alpha$ and $n s p$ are plotted in Fig. 7. As seen in Fig. 7, small angles at the singular vertex do not lead to poor accuracy whereas large angles that are proximal to $180^{\circ}$ are unfavorable. A combination of a large angle at the singular vertex and a small side of the triangle has the worst effect on the accuracy of the integration.

\section{Computational Fracture Simulations}

\subsection{Convergence study}

To demonstrate the effectiveness of the integration schemes, we first perform a convergence study over a cracked domain. Consider the cracked plate defined over the region $(-L, L) \times(-L, L)$ as shown in Fig. 8a with a crack that extends from $(-L, 0)$ to $(0,0)$. This problem is considered in Sukumar and Srolovitz. [72]. The material is assumed to be homogeneous and isotropic with $E=1$ and $\nu=0.3$. For the essential boundary conditions, we impose the near-tip displacement field corresponding to $K_{I}=1$ and $K_{I I}=0$. As a measure of accuracy, the relative energy norm of the error is used:

$$
E_{r e l}=\frac{\left\|\mathbf{u}-\mathbf{u}^{h}\right\|_{E(\Omega)}}{\|\mathbf{u}\|_{E(\Omega)}}=\frac{\left(\int_{\Omega}\left(\varepsilon-\varepsilon^{h}\right)^{T} \mathbf{D}\left(\varepsilon-\varepsilon^{h}\right) d \Omega\right)^{1 / 2}}{\left(\int_{\Omega} \varepsilon^{T} \mathbf{D} \varepsilon d \Omega\right)^{1 / 2}}
$$

where $\mathbf{u}$ and $\mathbf{u}^{h}$ are the exact and finite element solutions for the displacement field, $\varepsilon$ and $\varepsilon^{h}$ are the exact and finite element solutions for the strain and $\mathbf{D}$ is the plane strain constitutive matrix. Two strategies are chosen 


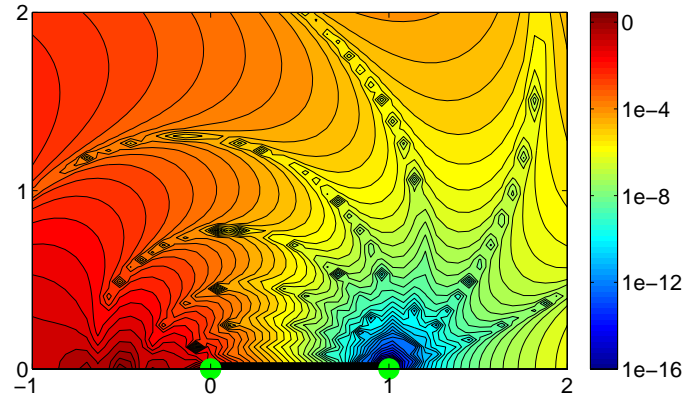

(a)

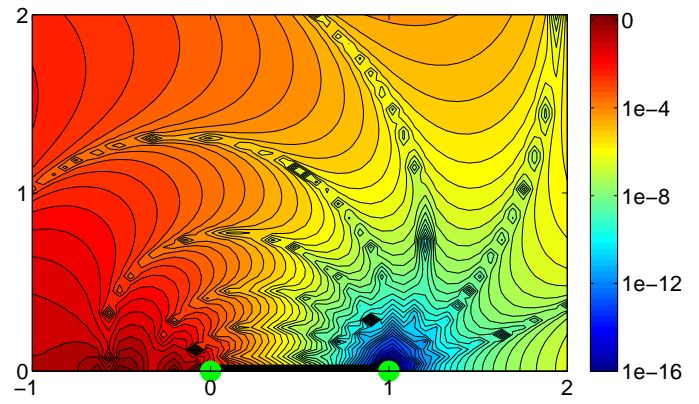

(c)

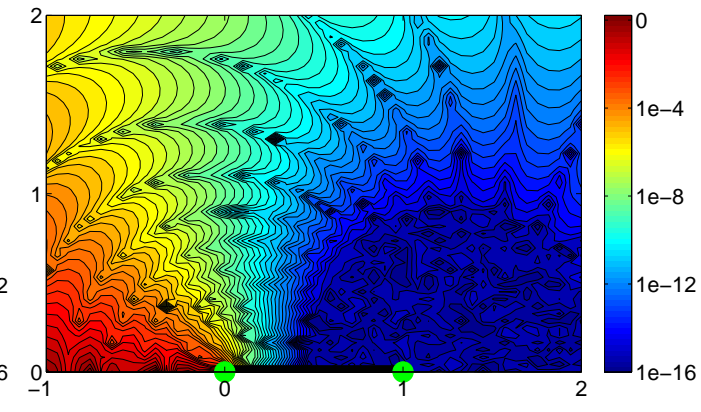

(b)

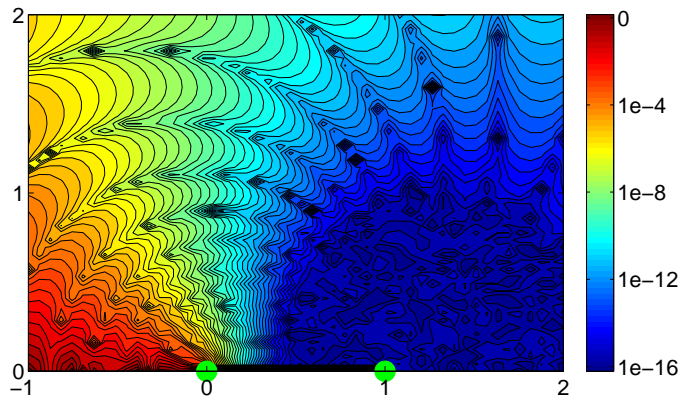

(d)

Fig. 7: Contour plots of the relative error in the evaluation of $\int_{\Omega} 1 / r^{\alpha} d \mathbf{x}$. (a) $\alpha=1, n s p=4 \times 4$; (b) $\alpha=1, n s p=10 \times 10$; (c) $\alpha=1 / 2, n s p=4 \times 4$; and (d) $\alpha=1 / 2, n s p=10 \times 10$. 
for near-tip enrichment [54]: topological enrichment where only one layer of elements around the crack-tip is enriched with crack-tip functions; and geometrical enrichment where all the nodes within a fixed distance $r_{e}$ from the crack-tip are enriched. In each case, the integration over the elements containing the crack-tip is carried out by subdividing the element into triangles and using two different integration schemes on each subtriangle: $5 \times 5$ tensorproduct; and $5 \times 5$ generalized Duffy transformation. Figures $8 \mathrm{a}$ and $8 \mathrm{~b}$ show the enriched nodes and the convergence curves for topological enrichment. The finite element node at $(-L, 0)$ is duplicated so that the exact essential boundary condition can be realized. Both integration methods produce a rate of convergence of $1 / 2$ in the relative energy norm of the error, which is in agreement with finite element theory. The enriched nodes and convergence curves for geometrical enrichment with two different enrichment radii $\left(r_{e}=0.25\right.$ and $\left.r_{e}=0.5\right)$ are depicted in Figures $8 \mathrm{c}$ and $8 \mathrm{~d}$. With geometric enrichment, smaller approximation errors are expected, and hence better accuracy in the numerical integration is required. The integration using $5 \times 5$ tensor-product rule yields a rate of convergence of 0.85 and 0.76 for enrichment radii of $r_{e}=0.25$ and $r_{e}=0.5$, respectively, whereas more accurate integration with the $5 \times 5$ generalized Duffy transformation is realized, which delivers the optimal first-order rate of convergence in the energy norm.

\subsection{Stress intensity factor calculations}

To validate the integration schemes presented in this paper we apply them for the calculation of stress intensity factors (SIFs) in an infinite plate with an inclined center crack under biaxial loading (Fig. 9a). The exact values of stress intensity factors are given by Aliabadi et al. [73]:

$$
\begin{aligned}
K_{I} & =\left(\sigma_{2} \sin ^{2} \beta+\sigma_{1} \cos ^{2} \beta\right) \sqrt{\pi a} \\
K_{I I} & =\left(\sigma_{2}-\sigma_{1}\right) \sin \beta \cos \beta \sqrt{\pi a},
\end{aligned}
$$




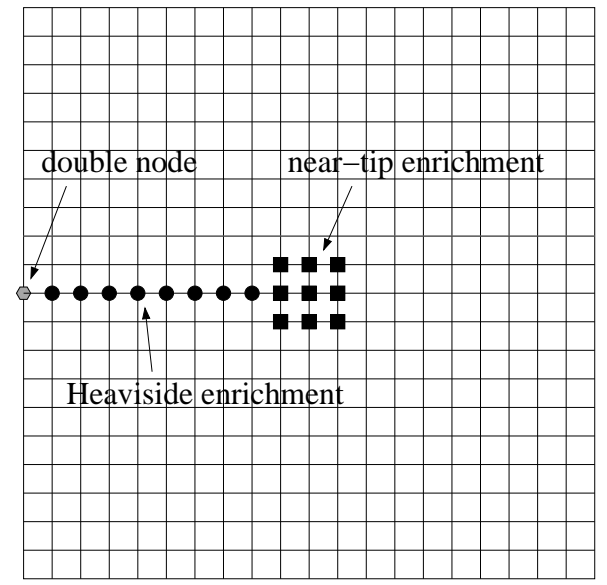

(a)

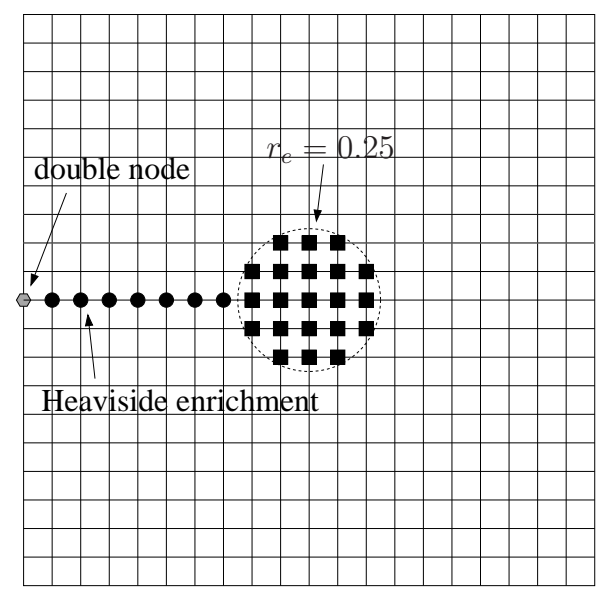

(c)

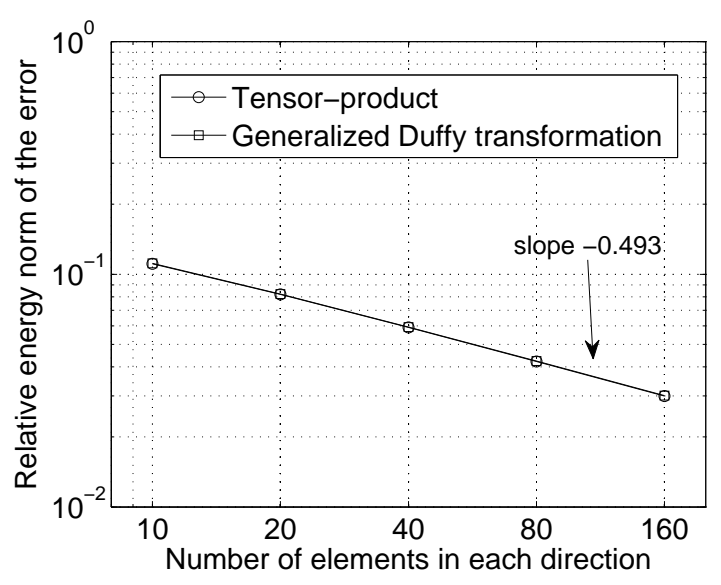

(b)

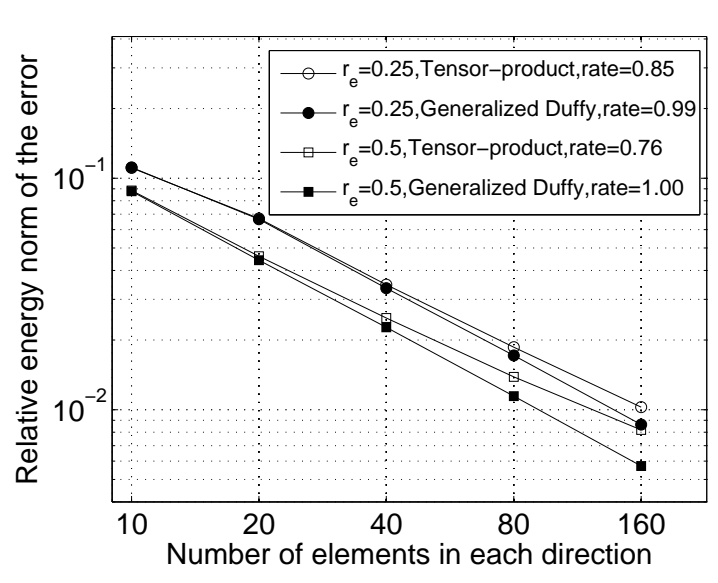

(d)

Fig. 8: Convergence study. (a),(b) enriched nodes for topological enrichment and relative energy norm of the error; and (c),(d) enriched nodes for geometrical enrichment (the case $r_{e}=0.25$ is depicted) and relative energy norm of the error. 
where $\sigma_{1}$ and $\sigma_{2}$ are the tensile loads in the $x_{1}$ - and $x_{2}$-directions, $a$ is the half-length of the crack, and $\beta$ is the inclination angle with respect to the vertical axis. The ratio of the length of the crack to the length of the plate $(a / w)$ is set to $1 / 10$ so that the problem domain can adequately represent an infinite domain. Numerical integration over the elements that do not have a discontinuity inside them, i.e., in the standard finite elements and in elements that are partially enriched for the Heaviside function is carried out with $2 \times 2$ tensor-product Gauss rule. For the elements with near-tip enrichment but not containing the crack-tip, $4 \times 4$ tensor product is used. We use our discontinuous quadratures for the elements that are fully cut by the crack and for the elements containing the crack-tip, $8 \times 8$ generalized Duffy transformation over the triangles is used. The results depicted in Fig. 9b and listed in Table 2 show a comparison of the exact SIFs from (12) and the ones calculated by the X-FEM on a $41 \times 41$ mesh. The X-FEM results show excellent agreement with the exact solution and the errors are similar to those obtained by Tabarraei and Sukumar [47]. The maximum relative error in $K_{I}$ and $K_{I I}$ is $0.6 \%$ and $2.4 \%$, respectively. Other benchmark problems such as an edge-crack under tension and shear loading $[5,74]$ have also been successfully tested using the proposed quadrature rules.

\subsection{Quasi-static crack growth}

\subsubsection{Edge-cracked plate in tension}

To show the effectiveness of the discontinuous quadrature rules, we use them for quasi-static crack growth simulations. Figure 10a shows a cracked domain with an inclined edge crack. The domain is the rectangle defined as $(0,1) \times(0,2)$ with a crack segment starting from $(0,1)$ and extending to $(0.4,1.4)$. The initial stress intensity factors calculated using the X-FEM on a $41 \times 81$ mesh are: $K_{I}=1.9060$ and $K_{I I}=0.8049$, which is in good agreement with the reference solution provided in Sutradhar et al. [75]. The 


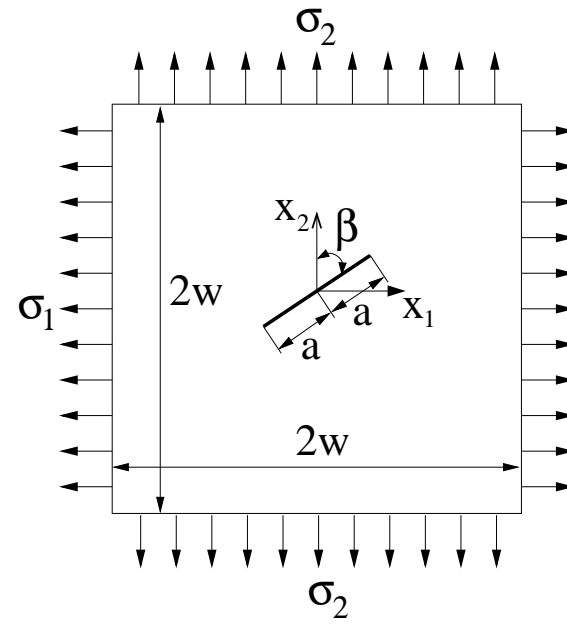

(a)

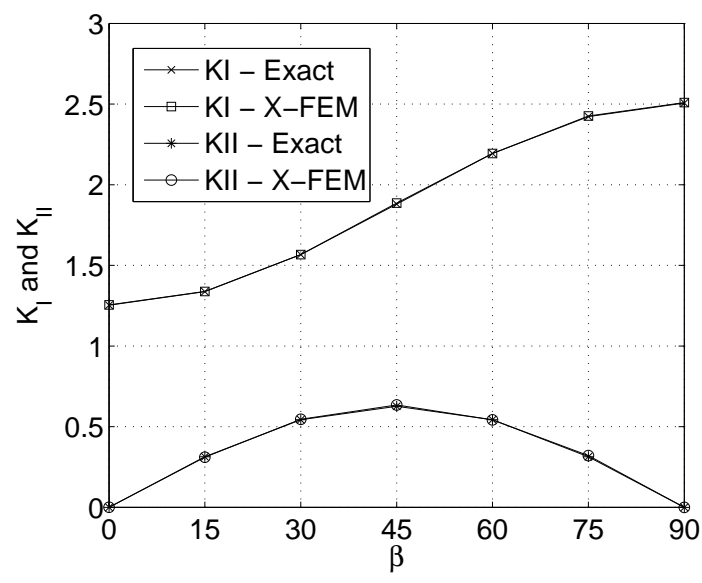

(b)

Fig. 9: SIF calculation for an inclined center-crack. (a) geometry; and (b) exact and extended finite element SIFs versus the inclination angle.

Table 2: Stress intensity factors for an infinite plate with an inclined center crack.

\begin{tabular}{ccccc}
\hline \multirow{2}{*}{$\beta$} & \multicolumn{2}{c}{ Exact } & \multicolumn{2}{c}{ X-FEM } \\
\cline { 2 - 5 } & $K_{I}$ & $K_{I I}$ & $K_{I}$ & $K_{I I}$ \\
\hline 0 & 1.2533 & 0.0000 & 1.2547 & 0.0000 \\
15 & 1.3373 & 0.3133 & 1.3379 & 0.3108 \\
30 & 1.5666 & 0.5427 & 1.5666 & 0.5457 \\
45 & 1.8800 & 0.6267 & 1.8864 & 0.6341 \\
60 & 2.1933 & 0.5427 & 2.1936 & 0.5421 \\
75 & 2.4227 & 0.3133 & 2.4255 & 0.3210 \\
90 & 2.5066 & 0.0000 & 2.5095 & 0.0000 \\
\hline
\end{tabular}


crack growth length in each step is assumed to be twice the size of the element containing the crack-tip and the maximum hoop (circumferential) stress criterion is used to find the crack growth angle. Figure 10b shows the crack growth path for meshes $41 \times 81$ and $81 \times 161$, and convergence in the crack path is observed.

To assess the efficiency of the discontinuous quadrature rules, we compare the total number of integration points over the elements that are cut by the crack, i.e., the elements with all their nodes enriched by the Heaviside function. Figure 11a shows the crack and the partitioned domain at the initial stage with 3-point standard quadrature rules over each of the triangles [69], whereas Fig. 11b shows the discontinuous quadrature rules over the same elements without partitioning them. At this stage, 720 integration points are required if partitioning is used compared to 105 evaluation points for the discontinuous quadrature rules. Figures 11c and $11 \mathrm{~d}$ exhibit similar trends at the final stage of crack growth: 1854 integration points are needed when partitioning is done and 195 points are required if the discontinuous quadrature rules are used. We point out that the discontinuous quadratures at each step of crack growth can be saved for the next step, and new discontinuous quadratures need to be constructed only over the elements at the crack-tip that were previously enriched with near-tip functions and are completely cut by the crack at the current step. The number of integration points of discontinuous quadrature rules vary from three over elements with simple geometries to five over elements with kinks inside them.

\subsubsection{Double-edge cracked plate in tension}

Finally, we consider quasi-static crack growth in a double-edge cracked plate in uniaxial tension. The geometry of the specimen with an initial crack is shown in Fig. 12a with parameters $a / w=0.1, h / w=2$, and $w=1$. The position of the edge cracks are perturbed in the vertical direction $(\delta=0.02)$ so 


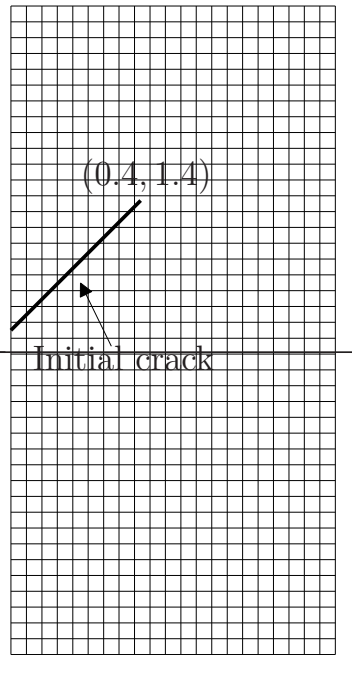

(a)

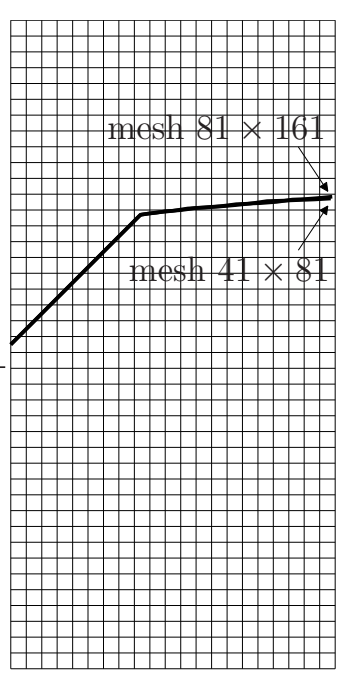

(b)

Fig. 10: Inclined crack growth problem. (a) initial crack; and (b) crack path.

that the cracks are under mixed-mode loading conditions. The crack growth procedure is identical to that followed in the previous problem (Section 4.3.1). Fig. 12b shows the crack path after 11 iterations. The integration points used for evaluation of stiffness matrix entries corresponding to the Heaviside enrichment are shown in Fig. 12c for standard quadrature rules over the triangle [69] (1806 integration points) and in Fig. 12d for the discontinuous quadratures (196 integration points).

\section{Concluding Remarks}

We applied the node elimination algorithm for the construction of efficient and accurate quadrature rules for fracture computations in the extended finite element method (X-FEM). For discontinuous functions, the quadrature rules obviated the need for partitioning and also resulted in significantly fewer integration points for evaluation of stiffness matrix entries. Construction of quadratic, cubic and quartic quadrature rules for integration of discontinuous functions over different polygons with arbitrary crack configurations 


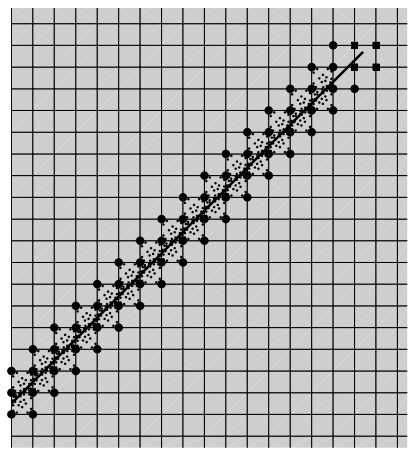

(a)

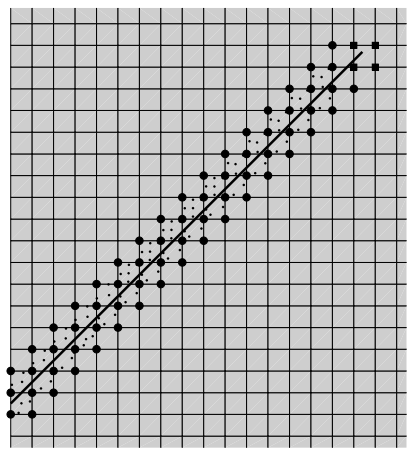

(b)

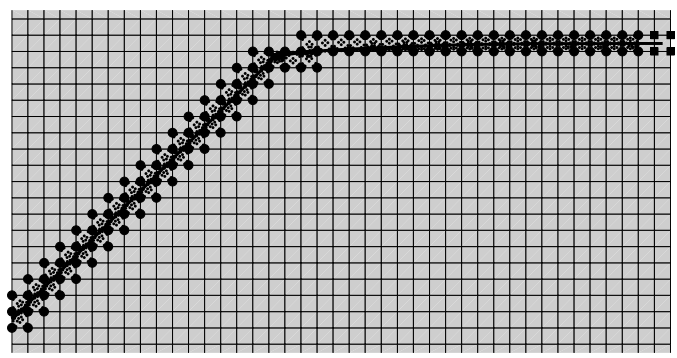

(c)

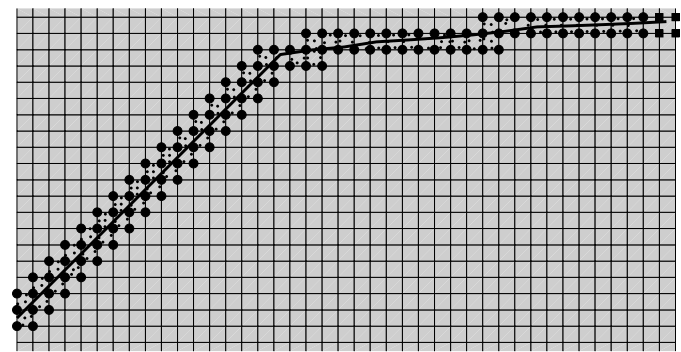

(d)

Fig. 11: Quadratures for integration of discontinuous functions. (a) initial stage, quadratures over partitions; (b) initial stage, discontinuous quadratures without partitioning; (c) final stage, quadratures over partitions; and (d) final stage, discontinuous quadratures without partitioning 


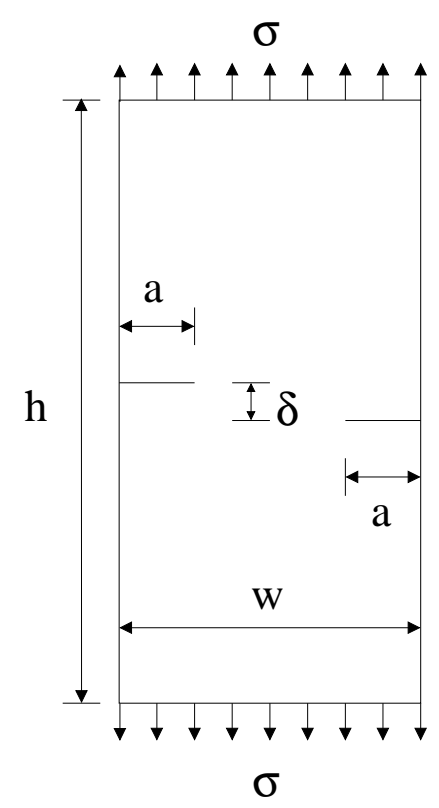

(a)

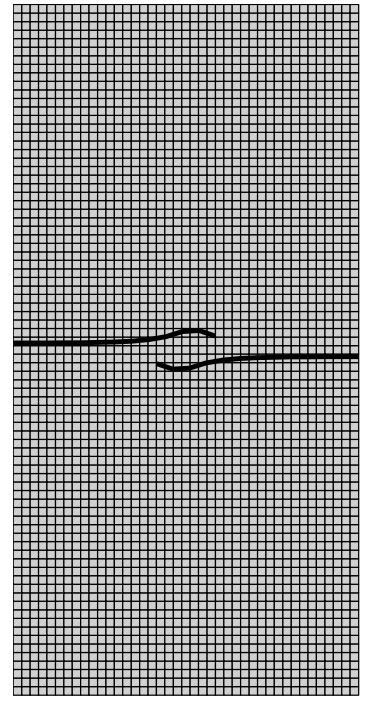

(b)

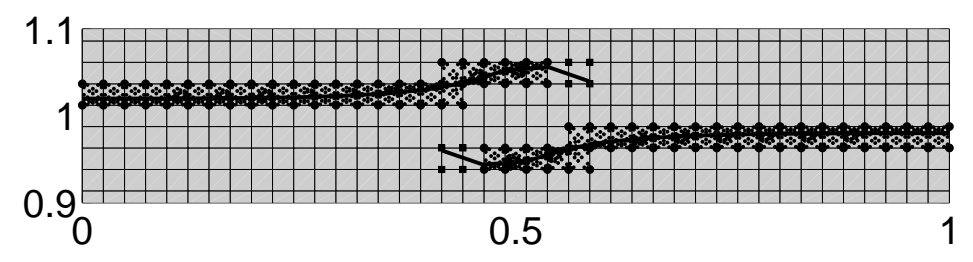

(c)

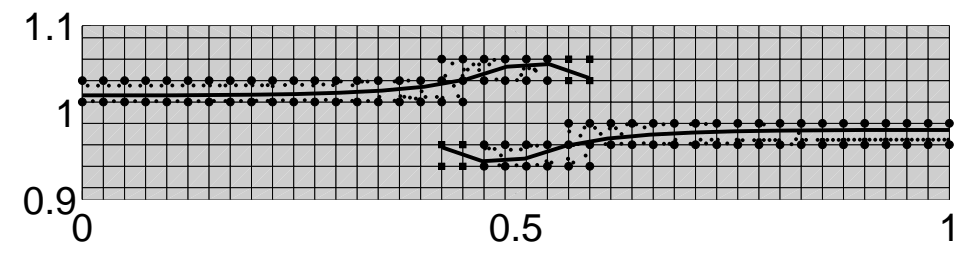

(d)

Fig. 12: Double-edge cracked plate in tension. (a) geometry; (b) crack path; (c) quadratures for integration of discontinuous functions over partitions (1806 integration points); and (d) quadratures for integration of discontinuous functions without partitioning (196 integration points). 
were presented. These calculations entail a one-time computational cost, and hence are of use in crack growth simulations.

The generalized Duffy transformation was adopted for the accurate integration of the derivatives of the near-tip enrichment functions within the X-FEM. The integration of functions with $1 / r$ and $1 / \sqrt{r}$ singularities over different arbitrary polygons and crack configurations were presented, which affirmed the markedly better performance of the generalized Duffy transformation vis-à-vis tensor-product rules and the Duffy transformation. A convergence study for an edge-crack using topological and geometrical enrichments was performed. The effectiveness of the generalized Duffy transformation in recovering the optimal first-order rate of convergence in the energy norm was shown. The discontinuous and singular quadratures were used in the benchmark problem of an inclined crack under biaxial tension-the mixed-mode stress intensity factors using the X-FEM were in good agreement with the exact solution, which provided further validation on the accuracy of the integration schemes. Finally, the merits of constructing discontinuous quadrature rules were demonstrated through two quasi-static crack growth simulations within the X-FEM-element-partitioning was not needed and in comparison to integration via partitioning, significantly fewer number of integration points were required. With suitable extensions of the algorithm, quadrature schemes can also be developed for higher-order finite elements and weak discontinuities [76-80].

\section{Acknowledgement}

The authors are grateful for the research support of the National Science Foundation through contract grants CMMI-0626481 and DMS-0811025 to the University of California at Davis. The authors thank Hong Xiao for her contributions in Reference [36], and the financial support from the UC Lab 
Fees Research Program is also acknowledged.

\section{References}

[1] C. A. Duarte, J. T. Oden, An h-p adaptive method using clouds, Computer Methods in Applied Mechanics and Engineering 139 (1996) 237262.

[2] J. M. Melenk, I. Babuška, The partition of unity finite element method: Basic theory and applications, Computer Methods in Applied Mechanics and Engineering 139 (1996) 289-314.

[3] I. Babuška, J. M. Melenk, The partition of unity method, International Journal for Numerical Methods in Engineering 40 (1997) 727-758.

[4] T. Belytschko, T. Black, Elastic crack growth in finite elements with minimal remeshing, International Journal for Numerical Methods in Engineering 45 (5) (1999) 601-620.

[5] N. Moës, J. Dolbow, T. Belytschko, A finite element method for crack growth without remeshing, International Journal for Numerical Methods in Engineering 46 (1) (1999) 131-150.

[6] J. Dolbow, N. Moës, T. Belytschko, Discontinuous enrichment in finite elements with a partition of unity method, Finite Elements in Analysis and Design 36 (2000) 235-260.

[7] C. Daux, N. Moës, J. Dolbow, N. Sukumar, T. Belytschko, Arbitrary branched and intersecting cracks with the extended finite element method, International Journal for Numerical Methods in Engineering 48 (12) (2000) 1741-1760. 
[8] T. Belytschko, N. Moës, S. Usui, C. Parimi, Arbitrary discontinuities in finite elements, International Journal for Numerical Methods in Engineering 50 (2001) 993-1013.

[9] A. Hansbo, P. Hansbo, A finite element method for the simulation of strong and weak discontinuities in solid mechanics, Computer Methods in Applied Mechanics and Engineering 193 (33-35) (2004) 3523-3540.

[10] T. P. Fries, T. Belytschko, The intrinsic XFEM: a method for arbitrary discontinuities without additional unknowns, International Journal for Numerical Methods in Engineering 68 (2006) 1358-1385.

[11] E. Chahine, P. Laborde, Y. Renard, Crack tip enrichment in the XFEM using a cutoff function, International Journal for Numerical Methods in Engineering 75 (2008) 629-646.

[12] Y. Shen, A. Lew, An optimally convergent discontinuous-Galerkin-based extended finite element method for fracture mechanics, International Journal for Numerical Methods in Engineering 82 (6) (2010) 716-755.

[13] S. E. Mousavi, N. Sukumar, Generalized Duffy transformation for integrating vertex singularities, Computational Mechanics 45 (2-3) (2010) $127-140$.

[14] T. Belytschko, H. Chen, J. Xu, G. Zi, Dynamic crack propagation based on loss of hyperbolicity and a new discontinuous enrichment, International Journal for Numerical Methods in Engineering 58 (2003) 18731905.

[15] J. Réthoré, A. Gravouil, A. Combescure, An energy-conserving scheme for dynamic crack growth using the extended finite element method, International Journal for Numerical Methods in Engineering 63 (2005) $631-659$. 
[16] B. Prabel, A. Combescure, A. Gravouil, S. Marie, Level set X-FEM non-matching meshes: Application to dynamic crack propagation in elastic-plastic media, International Journal for Numerical Methods in Engineering 69 (2007) 1553-1569.

[17] G. N. Wells, L. J. Sluys, A new method for modelling cohesive cracks using finite elements, International Journal for Numerical Methods in Engineering 50 (2001) 2667-2682.

[18] Moës, T. Belytschko, Extended finite element method for cohesive crack growth, Engineering Fracture Mechanics 69 (2002) 813-833.

[19] J. Mergheim, E. Kuhl, P. Steinmann, A finite element method for the computational modelling of cohesive cracks, International Journal for Numerical Methods in Engineering 63 (2005) 276-289.

[20] S. Gross, A. Reusken, An extended pressure finite element space for two-phase incompressible flows with surface tension, Journal of Computational Physics 224 (2007) 40-58.

[21] A. Zilian, A. Legay, The enriched space-time finite element method (EST) for simultaneous solution of fluid-structure interaction, International Journal for Numerical Methods in Engineering 75 (2008) 305-334.

[22] A. Gerstenberger, W. A. Wall, An extended finite element method/Lagrange multiplier based approach for fluid-structure interaction, Computer Methods in Applied Mechanics and Engineering 197 (2008) 1699-1714.

[23] T. P. Fries, The intrinsic XFEM for two-fluid flows, International Journal for Numerical Methods in Engineering 60 (2009) 437-471. 
[24] L. M. Vigneron, J. G. Verly, S. K. Warfield, Modelling surgical cuts, retractions, and resections via extended finite element method, in: Barillot, $\mathrm{C}$ and Haynor, DR and Hellier, P (Ed.), Medical image computing and computer assisted intervention - MICCAI 2004, PT 2, Proceedings, Vol. 3217 (Part 2) of Lecture Notes in Computer Science, SpringerVerlag Berlin, Heidelberger Platz 3, D-14197 Berlin, Germany, 2004, pp. $311-318$.

[25] L. Jeřábková, T. Kuhlen, Stable cutting of deformable objects in virtual environments using the XFEM, IEEE Computer Graphics and Applications 29 (2) (2009) 61-71.

[26] P. Kaufmann, S. Martin, M. Botsch, E. Grinspun, M. Gross, Enrichment textures for detailed cutting of shells, ACM Transactions on Graphics 28 (3) (2009) 50:1-50:10.

[27] G. Ventura, On the elimination of quadrature subcells for discontinuous functions in the extended finite-element method, International Journal for Numerical Methods in Engineering 66 (2006) 761-795.

[28] A.-K. Tornberg, Multi-dimensional quadrature of singular and discontinuous functions, BIT Numerical Mathematics 42 (3) (2002) 644-669.

[29] B. Patzák, M. Jirásek, Process zone resolution by extended finite elements, Engineering Fracture Mechanics 70 (2003) 957-977.

[30] H. Oh, J. G. Kim, J. W. Jeong, The smooth piecewise polynomial particle shape functions corresponding to patch-wise non-uniformly spaced particles for meshfree particle methods, Computational Mechanics 40 (2007) 569-594.

[31] E. Benvenuti, A. Tralli, G. Ventura, A regularized XFEM model for the 
transition from continuous to discontinuous displacements, International Journal for Numerical Methods in Engineering 74 (2008) 911-944.

[32] S. Bordas, P. V. Nguyen, C. D. A. Guidoum, H. Nguyen-Dang, An extended finite element library, International Journal for Numerical Methods in Engineering 71 (2007) 703-732.

[33] D. Holdych, D. Noble, R. Secor, Quadrature rules for triangular and tetrahedral elements with generalized functions, International Journal for Numerical Methods in Engineering 73 (2008) 1310-1327.

[34] T. Belytschko, R. Gracie, G. Ventura, A review of extended/generalized finite element methods for material modeling, Modelling and Simulation in Materials Science and Engineering 17 (4).

[35] H. Xiao, Z. Gimbutas, A numerical algorithm for the construction of efficient quadratures in two and higher dimensions, Computers and Mathematics with Applications 59 (2010) 663-676.

[36] S. E. Mousavi, H. Xiao, N. Sukumar, Generalized Gaussian quadrature rules on arbitrary polygons, International Journal for Numerical Methods in Engineering 82 (2010) 99-113.

[37] R. S. Barsoum, Application of quadratic isoparametric elements in linear fracture mechanics, International Journal of Fracture 10 (1974) 603-605.

[38] M. Stern, E. B. Becker, A conforming crack tip element with quadratic variation in the singular fields, International Journal for Numerical Methods in Engineering 12 (1978) 279-288.

[39] D. M. Tracey, Finite elements for determination of crack tip elastic stress intensity factors, Engineering Fracture Mechanics 3 (1971) 255-265. 
[40] J. S. Solecki, J. L. Swedlow, On quadrature and singular finite-elements, International Journal for Numerical Methods in Engineering 20 (1984) $395-408$.

[41] J. E. Akin, The generation of elements with singularities, International Journal for Numerical Methods in Engineering 10 (1976) 1249-1259.

[42] D. M. Tracey, T. S. Cook, Analysis of power type singularities using finite elemetns, International Journal for Numerical Methods in Engineering 11 (1977) 1225-1233.

[43] I. L. Lim, I. W. Johnston, S. K. Choi, Application of singular quadratic distorted isoparametric elements in linear fracture mechanics, International Journal for Numerical Methods in Engineering 36 (1993) 24732499 .

[44] R. S. Dunham, A quadrature rule for conforming quadratic crack tip elements, International Journal for Numerical Methods in Engineering 14 (1979) 287-312.

[45] S. Natarajan, S. Bordas, D. R. Mahapatra, Numerical integration over arbitrary polygonal domains based on Schwarz-Christoffel conformal mapping, International Journal for Numerical Methods in Engineering 80 (2009) 103-134.

[46] N. Sukumar, A. Tabarraei, Conforming polygonal finite elements, International Journal for Numerical Methods in Engineering 61 (12) (2004) 2045-2066.

[47] A. Tabarraei, N. Sukumar, Extended finite element method on polygonal and quadtree meshes, Computer Methods in Applied Mechanics and Engineering 197 (5) (2008) 425-438. 
[48] W. Gander, W. Gautschi, Adaptive quadrature revisited, BIT Numerical Mathematics 40 (1) (2004) 84-101.

[49] T. Strouboulis, I. Babuška, K. Copps, The design and analysis of the generalized finite element method, Computer Methods in Applied Mechanics and Engineering 181 (1-3) (2000) 43-69.

[50] Q. Z. Xiao, B. L. Karihaloo, Improving the accuracy of XFEM crack tip fields using higher order quadrature and statically admissible stress recovery, International Journal for Numerical Methods in Engineering 66 (2006) 1378-1410.

[51] T. O. Espelid, A. Genz, DECUHR: an algorithm for automatic integration of singular functions over a hyperrectangular region, Numerical Algorithms 8 (1994) 201-220.

[52] M. A. Schweitzer, An adaptive hp-version of the multilevel particlepartition of unity method, Computer Methods in Applied Mechanics and Engineering 198 (2009) 1260-1272.

[53] M. Griebel, M. A. Schweitzer, A particle-partition of unity methodPart II: Efficient cover construction and reliable integration, SIAM Journal on Scientific Computing 23 (5) (2002) 1655-1682.

[54] E. Béchet, H. Minnebo, N. Moës, B. Burgardt, Improved implementation and robustness study of the X-FEM for stress analysis around cracks, International Journal for Numerical Methods in Engineering 64 (8) (2005) 1033-1056.

[55] K. Park, J. P. Pereira, C. A. Duarte, G. H. Paulino, Integration of singular enrichment functions in the generalized/extended finite element method for three-dimensional problems, International Journal for $\mathrm{Nu}-$ merical Methods in Engineering 78 (10) (2009) 1220-1257. 
[56] A. Nagarajan, S. Mukherjee, A mapping method for numerical evaluation of two-dimensional integrals with $1 / r$ singularity, Computational Mechanics 12 (1993) 19-26.

[57] G. Ventura, R. Gracie, T. Belytschko, Fast integration and weight function blending in the extended finite element method, International Journal for Numerical Methods in Engineering 77 (1) (2009) 1-29.

[58] P. Laborde, J. Pommier, Y. Renard, M. Salaün, High-order extended finite element method for cracked domains, International Journal for Numerical Methods in Engineering 64 (2005) 354-381.

[59] G. Fairweather, F. J. Rizzo, D. J. Shippy, Computation of double integrals in the boundary integral equation method, in: R. Vichnevetsky, R. S. Stepleman (Eds.), Advances in Computer Methods for Partial Differential Equations - III, IMACS Publ., Brussels, Belgium, 1979, pp. $331-334$.

[60] M. G. Duffy, Quadrature over a pyramid or cube of integrands with a singularity at a vertex, SIAM Journal on Numerical Analysis 19 (6) (1982) 1260-1262.

[61] J. N. Lyness, D. Jespersen, Moderate degree symmetric quadrature rules for the triangle, Journal of the Institute of Mathematics and its Applications 15 (1975) 19-32.

[62] M. Nooijen, G. T. Velde, E. J. Baerends, Symmetric numerical integration formulas for regular polygons, SIAM Journal on Numerical Analysis 27 (1) (1990) 198-218.

[63] S. Wandzura, H. Xiao, Symmetric quadrature rules on a triangle, Computers and Mathematics with Applications 45 (2003) 1829-1840. 
[64] N. Sukumar, J.-H. Prévost, Modeling quasi-static crack growth with the extended finite element method. Part I: Computer implementation, International Journal of Solids and Structures 40 (26) (2003) 7513-7537.

[65] J. B. Lasserre, Integration on a convex polytope, Proceedings of the American Mathematical Society 126 (8) (1998) 2433-2441.

[66] J. B. Lasserre, Integration and homogeneous functions, Proceedings of the American Mathematical Society 127 (3) (1999) 813-818.

[67] N. Sukumar, D. J. Srolovitz, T. J. Baker, J.-H. Prévost, Brittle fracture in polycrystalline microstructures with the extended finite element method, International Journal for Numerical Methods in Engineering 56 (14) (2003) 2015-2037.

[68] A. Simone, C. A. Duarte, E. Van der Giessen, A generalized finite element method for polycrystals with discontinuous grain boundaries, International Journal for Numerical Methods in Engineering 67 (8) (2006) $1122-1145$.

[69] D. Dunavant, High degree efficient symmetrical Gaussian quadrature rules for the triangle, International Journal for Numerical Methods in Engineering 21 (1985) 1129-1148.

[70] A. Haegemans, Cubature formulas for triangles and squares with a $1 / r$ singularity, Tech. Rep. TW 192, Department of Computer Science, K. U. Leuven, Belgium (1993).

[71] J. N. Lyness, On handling singularities in finite elements, in: T. O. Espelid, A. Genz (Eds.), Numerical Integration, Recent Developments, Software and Applications, NATO ASI Series C: Mathematical and Physical Sciences, Vol. 357, Kluwer Academic Publishers, Dordrecht, The Netherlands, 1992, pp. 219-233. 
[72] N. Sukumar, D. J. Srolovitz, Finite element-based model for crack propagation in polycrystalline materials, Computational and Applied Mathematics 23 (2004) 363-380.

[73] M. H. Aliabadi, D. P. Rooke, D. J. Cartwright, Mixed-mode Bueckner weight functions using boundary element analysis, International Journal of Fracture 34 (1987) 131-147.

[74] F. L. Stazi, E. Budyn, J. Chessa, T. Belytschko, An extended finite element method with higher-order elements for curved cracks, Computational Mechanics 31 (2003) 38-48.

[75] A. Sutradhar, G. H. Paulino, L. J. Gray, Symmetric Galerkin Boundary Element Method, Springer-Verlag, Berlin Heidelberg, 2008.

[76] N. Sukumar, D. L. Chopp, N. Moës, T. Belytschko, Modeling holes and inclusions by level sets in the extended finite-element method, Computer Methods in Applied Mechanics and Engineering 190 (46-47) (2001) 6183-6200.

[77] N. Moës, M. Cloirec, P. Cartraud, J.-F. Remacle, A computational approach to handle complex microstructure geometries, Computer Methods in Applied Mechanics and Engineering 192 (2003) 3163-3177.

[78] J. Chessa, T. Belytschko, An enriched finite element method and level sets for axisymmetric two-phase flow with surface tension, International Journal for Numerical Methods in Engineering 58 (2003) 2041-2064.

[79] A. Legay, H. W. Wang, T. Belytschko, Strong and weak arbitrary discontinuities in spectral finite elements, International Journal for Numerical Methods in Engineering 64 (2005) 991-1008. 
[80] S. Zlotnik, P. Díez, Hierarchical X-FEM for $n$-phase flow $(n>2)$, Computer Methods in Applied Mechanics and Engineering 198 (2009) 2329 2338 . 